\title{
THE EVOLUTION OF HELIUM SHELL-BURNING STARS
}

\author{
D. J. FAULKNER \\ (Mount Stromlo Observatory, Australia)
}

Evolutionary sequences of stellar models have been obtained, using the Henyey method, for stars of mass $0.8 M_{\odot}, 0.9 M_{\odot}$ and $1.0 M_{\odot}$. The starting-point of each sequence is a star with a core of carbon and oxygen, comprising $25 \%$ of the star's mass, surrounded by helium-rich material. It is assumed that any remaining hydrogenrich material has been ejected in the form of the planetary shell. The star contracts gravitationally until helium ignites in a shell at the composition discontinuity, and the subsequent evolution of the shell burning has been followed. No neutrino-loss processes have been considered in this investigation.

As the evolution proceeds, the shell becomes thinner, but an increase in its temperature outweighs this effect and the resultant energy production, and hence the luminosity of the star, increases sharply. The radial distance to the shell decreases slightly, but the exterior layers of the star expand at first, and the total radius increases. In the case of the $1.0 M_{\odot}$ sequence this expansion is quite considerable. The radius of the star passes through a maximum and decreases again. Eventually, the contraction of the region interior to the shell source becomes insufficient to maintain the rate of energy production in the source, and the luminosity, too, passes through a maximum. The temperature in the core becomes high enough for carbon burning to commence, and when this occurs, the layers immediately surrounding the core expand and the helium-burning shell dies out. The star then settles down to a phase of carbon burning in a convective core. The present evolutionary sequences have been terminated at this point.

The latter parts of the computed tracks in the Hertzsprung-Russell diagram match the observed track for the nuclei of planetary nebulae; the computed times of evolution are longer than the observed time by a factor of 5-10. They are, however, considerably shorter than the evolution times obtained (in the absence of neutrino processes) for stars without shell burning, which are too long by two orders of magnitude (Vila, Astrophys. J., 146, 1966, 437). It thus appears that the inclusion of shell burning will render less sharp any test of the existence of the universal Fermi interaction based on a comparison of the observed and computed evolution times of the nuclei of planetary nebulae.

The present results suggest that the planetary nebula phase of a star's evolution may occur between helium burning and carbon burning, rather than directly before the white-dwarf stage. A more detailed description of this work will appear elsewhere (Mon. Not. R. astr. Soc., in press). 